\title{
Thimble regularization at work for Gauge Theories: from toy models onwards.
}

\author{
Francesco Di Renzo* \\ University of Parma and INFN \\ E-mail: francesco.direnzo@unipr.it
}

\section{Giovanni Eruzzi}

Università di Parma and INFN

E-mail: giovanni.eruzziefis.unipr.it

\begin{abstract}
A final goal for thimble regularization of lattice field theories is the application to lattice QCD and the study of its phase diagram. Gauge theories pose a number of conceptual and algorithmic problems, some of which can be addressed even in the framework of toy models. We report on our progresses in this field, starting in particular from first successes in the study of one link models.
\end{abstract}

The 33rd International Symposium on Lattice Field Theory

14 -18 July 2015

Kobe International Conference Center, Kobe, Japan

${ }^{*}$ Speaker. 


\section{Thimble regularization in a nutshell}

Thimble regularization has been proposed in the broad context of extending our capabilities to properly define quantum field theories [1]. It has been later applied to lattice field theories as an attempt to overcome the sign problem [2,3]. In a nutshell, it relies on the complexification of the original degrees of freedom. The functional integral is defined on manifolds which roughly speaking emerge as the generalization of Steepest Descent (SD) paths. Not surprisingly, such an approach displays the same virtues of saddle point evaluation of integrals, i.e. stationary phase and localization of important contributions.

Morse theory [4] states that under suitable (but not so strict) conditions on holomorphic functions $S(x)=S_{R}+i S_{I}$ and $O(x)$

$$
\int_{\mathscr{C}} d x O(x) \mathrm{e}^{-S(x)}=\sum_{\sigma} n_{\sigma} \mathrm{e}^{-i S_{I}\left(p_{\sigma}\right)} \int_{\mathscr{J}_{\sigma}} d z O(z) \mathrm{e}^{-S_{R}(z)}
$$

where the notation $(S, O)$ alludes to a functional integral (even if there is no normalizing factor $Z^{-1}$ ). The content of (1.1) has to be understood as follows:

- the greek index $\sigma$ counts the stationary points $p_{\sigma}$ of the complex function $S(z)$;

- each (stable) thimble $\mathscr{J}_{\sigma}$ is the union of all the Steepest Ascent (SA) paths falling into $p_{\sigma}$ at (minus) infinite time, i.e. the union of the solutions of

$$
\begin{aligned}
\frac{d x_{i}}{d \tau} & =\frac{\partial S_{R}(x, y)}{\partial x_{i}} \\
\frac{d y_{i}}{d \tau} & =\frac{\partial S_{R}(x, y)}{\partial y_{i}}
\end{aligned}
$$

satisfying $z(\tau=-\infty)=x(\tau=-\infty)+i y(\tau=-\infty)=p_{\sigma}$

- in the homological sense $\mathscr{C}=\sum_{\sigma} n_{\sigma} \mathscr{J}_{\sigma}$ and the thimbles have the same real dimension of the original domain of integration;

- the coefficients $n_{\sigma}$ count the intersections of the unstable thimbles $\mathscr{K}_{\sigma}$ with the original domain of integration; unstable thimbles are solutions of (1.2) with $z(\tau=\infty)=p_{\sigma}$.

- the imaginary part $S_{I}$ stays constant on a thimble; on the other side $S_{R}$ increases along the SA solutions of (1.2), thus ensuring convergence of (1.1).

For the following it is useful to remind the reader of a constructive approach. Near a critical point a field configuration can be expressed as $\Phi_{i}=\phi_{i}-\phi_{\sigma, i}$; the real part of the action is in turn

$$
S_{R}(\phi)=S_{R}\left(\phi_{\sigma}\right)+\frac{1}{2} \Phi^{T} H \Phi+\mathscr{O}\left(\phi^{3}\right)
$$

where $H$ is the hessian

$$
H_{i j}=\left.\frac{\delta^{2} S_{R}}{\delta \phi_{i} \delta \phi_{j}}\right|_{\phi=\phi_{\sigma}}
$$


which can be put in diagonal form

$$
H=W \Lambda W^{T} \quad \Lambda=\operatorname{diag}\left(\lambda_{1}, \cdots, \lambda_{n},-\lambda_{1}, \cdots,-\lambda_{n}\right)
$$

once its eigenvalues are known and arranged in the matrix $W$. The reader should notice the form of the spectrum: there is an equal number of positive and negative eigenvalues. Half of the eigenvectors of the Hessian (those corresponding to positive eigenvalues) span the tangent space at the stable thimble at the critical point: if one leaves the critical point along those directions integrating the SA equations, one covers the stable thimble. On the other side, the other directions take you along the unstable thimble.

At a generic point we lack an a priori knowledge of the tangent space. The (local) basis can be nevertheless obtained by transporting along the flow the basis at the critical point (see [2] or [3] for details). By doing this one also realizes that the relative orientation between the canonical complex volume form and the real volume form, characterizing the tangent space of the thimble, contributes a phase to the integral. This is termed the residual phase (see [5] for details).

\section{Thimble regularization for gauge theories}

\subsection{The basic set-up}

We now want to sketch the thimble construction for $\mathrm{SU}(N)$ gauge theories defined by an action $S[U]$. Going to complex fields means

$$
\mathrm{SU}(N) \ni U=e^{i x_{a} T^{a}} \rightarrow e^{i z_{a} T^{a}}=e^{i\left(x_{a}+i y_{a}\right) T^{a}} \in \operatorname{SL}(N, \mathbb{C})
$$

with the caveat that

$$
\mathrm{SU}(N) \ni U^{\dagger}=e^{-i x_{a} T^{a}} \rightarrow e^{-i z_{a} T^{a}}=e^{-i\left(x_{a}+i y_{a}\right) T^{a}}=U^{-1} \in \mathrm{SL}(N, \mathbb{C}) .
$$

Main ingredient is the Lie derivative

$$
\nabla^{a} f(U)=\lim _{\alpha \rightarrow 0} \frac{1}{\alpha}\left[f\left(e^{i \alpha T^{a}} U\right)-f(U)\right]=\left.\frac{\delta}{\delta \alpha} f\left(e^{i \alpha T^{a}} U\right)\right|_{\alpha=0}
$$

in terms of which we can write the SA equations as

$$
\frac{\mathrm{d}}{\mathrm{d} \tau} U_{\hat{\mu}}(n ; \tau)=\left(i T^{a} \bar{\nabla}_{n, \hat{\mu}}^{a} \overline{S[U(\tau)]}\right) U_{\hat{\mu}}(n ; \tau) .
$$

The solutions of these equations display the main properties we expect. Namely, since $\frac{\mathrm{d}}{\mathrm{d} \tau}=$ $\bar{\nabla}_{n, \hat{\mu}}^{a} \bar{S} \nabla_{n, \hat{\mu}}^{a}+\nabla_{n, \hat{\mu}}^{a} S \bar{\nabla}_{n, \hat{\mu}}^{a}$ we have that

$$
\frac{\mathrm{d} S^{R}}{\mathrm{~d} \tau}=\frac{1}{2} \frac{\mathrm{d}}{\mathrm{d} \tau}(S+\bar{S})=\frac{1}{2}\left(\bar{\nabla}_{n, \hat{\mu}}^{a} \bar{S} \nabla_{n, \hat{\mu}}^{a} S+\nabla_{n, \hat{\mu}}^{a} S \bar{\nabla}_{n, \hat{\mu}}^{a} \bar{S}\right)=\|\nabla S\|^{2} \geq 0
$$

and

$$
\frac{\mathrm{d} S^{I}}{\mathrm{~d} \tau}=\frac{1}{2 i} \frac{\mathrm{d}}{\mathrm{d} \tau}(S-\bar{S})=\frac{1}{2 i}\left(\bar{\nabla}_{n, \hat{\mu}}^{a} \bar{S} \nabla_{n, \hat{\mu}}^{a} S-\nabla_{n, \hat{\mu}}^{a} S \bar{\nabla}_{n, \hat{\mu}}^{a} \bar{S}\right)=0 .
$$


Lie derivatives obey non-trivial commutation relations

$$
\begin{aligned}
& {\left[\nabla_{n, \hat{\mu}}^{a}, \nabla_{m, \hat{v}}^{b}\right]=-f^{a b c} \nabla_{n, \hat{\mu}}^{c} \delta_{n, m} \delta_{\hat{\mu}, \hat{v}}} \\
& {\left[\bar{\nabla}_{n, \hat{\mu}}^{a}, \bar{\nabla}_{m, \hat{v}}^{b}\right]=-f^{a b c} \bar{\nabla}_{n, \hat{\mu}}^{c} \delta_{n, m} \delta_{\hat{\mu}, \hat{v}}} \\
& {\left[\nabla_{n, \hat{\mu}}^{a}, \bar{\nabla}_{m, \hat{v}}^{b}\right]=0}
\end{aligned}
$$

from which we can get commutation relations for vectors $V \equiv V_{n, \hat{\mu}, a} \nabla_{n, \hat{\mu}}^{a}+\bar{V}_{n, \hat{\mu}, a} \bar{\nabla}_{n, \hat{\mu}}^{a}$

$$
\left[V, V^{\prime}\right]_{n, \hat{\mu}, c}=-f^{a b c} V_{n, \hat{\mu}, a} V_{n, \hat{\mu}, b}^{\prime} .
$$

Taking $V_{n, \hat{\mu}, c}^{\prime}=\bar{\nabla}_{n, \hat{\mu}}^{c} \bar{S}$ we can derive the equation for transporting a vector $V$ from the critical point to any point along the flow described by (2.1)

$$
\frac{\mathrm{d}}{\mathrm{d} \tau} V_{n, \hat{\mu}, c}=\bar{\nabla}_{m, \hat{v}}^{a} \bar{\nabla}_{n, \hat{\mu}}^{c} \bar{S} \bar{V}_{m, \hat{v}, a}+f^{a b c} \bar{\nabla}_{n, \hat{\mu}}^{b} \bar{S} V_{n, \hat{\mu}, a} .
$$

Apparently we collected all the tools needed for the constructive approach to thimbles we described in the previous section. In particular, the last equation we wrote would enable us to transport along the flow the basis of the tangent space at the critical point. As a matter of fact all this is still void, because we still miss a proper definition for thimbles. While till now everything seems to be quite natural, we soon realize we need new ingredients to generalize the construction of thimbles in the case of gauge theories.

\subsection{From non-degenerate critical points to non-degenerate critical submanifolds}

Once local gauge invariance is in place, every stationary point of a gauge-invariant action belongs to a manifold of stationary points and, in particular, the Hessian is degenerate. The relevant picture is now provided [6] by generalizing the concept of a non-degenerate critical point ${ }^{1}$ into that of a non-degenerate critical manifold [6]. A manifold $\mathscr{N} \subset \mathscr{C}$ is a non-degenerate critical submanifold of $\mathscr{C}$ for the function $F: \mathscr{C} \rightarrow \mathbb{R}$ if:

1. $d F=0$ along $\mathscr{N}$;

2. The Hessian $\partial^{2} F$ is non-degenerate on the normal bundle $v(\mathscr{N})$.

If we consider the $A=0$ vacuum of an $S U\left(N_{c}\right)$ Yang-Mills theory ( $F$ being given by the action $S$ ), $\mathscr{N}^{(0)}$ is given by the complete gauge orbit associated to $A=0$, the real dimension of such critical sub-manifold being given by $(V-1)\left(N_{c}^{2}-1\right)$.

When we complexify, we switch to $S L\left(N_{c}, \mathbb{C}\right)$ and we get instead the gauge orbit in the latter. As for non-zero eigenvalues of the action hessian, we get an equal number of positive and negative eigenvalues. These are once again associated to the SA and SD flows described by (2.1) (and we will be once again left with the right real dimension of the thimbles).

All in all, the thimble e.g. associated to $A=0$ for the $S U(3)$ Yang-Mills action is defined by $\mathscr{J}_{0}:=\left\{U \in(S L(3, \mathbb{C}))^{4 V} \mid \exists U(\tau)\right.$ solution of Eq. (2.1) $\left.\mid U(0)=U \& \lim _{\tau \rightarrow-\infty} U(\tau) \in \mathscr{N}^{(0)}\right\}$.

\footnotetext{
${ }^{1}$ The stationary points of Section 1 were non-degenerate critical points: the hessian had no zero eigenvalue.
} 
The meaning of the construction we sketched gets clearer when one realizes that under $S L(3, \mathbb{C})$ gauge transformations $U_{v}(x) \rightarrow \Lambda(x) U_{v}(x ; \tau) \Lambda(x+\hat{v})^{-1}$

$$
\left(T_{a} \bar{\nabla}_{x, v, a} \overline{S[U]}\right) \rightarrow\left(\Lambda(x)^{-1}\right)^{\dagger}\left(T_{a} \bar{\nabla}_{x, v, a} \overline{S[U]}\right) \Lambda(x)^{\dagger} .
$$

This means that we have the SA covariant only provided $\Lambda(x)^{\dagger}=\Lambda(x)^{-1}$, i.e. for $S U(3)$ transformations. This also means that if we take a SA from $A=0$, at any stage we can perform a gauge transformation and this will take us to a point starting from which under SD we are going to eventually land on another point on the gauge orbit of $A=0$ (decided by the gauge transformation we choose). For further details on the construction we sketched the reader is referred to [2].

\subsection{Pure Yang Mills SU(N): torons and all that}

Thimble regularization is per se an interesting subject. Nevertheless, the main motivation for probing it as a solution of the sign problem is to eventually tackle the investigation of the QCD phase diagram. It is thus of outmost importance that the framework we have just sketched is proven to be effective for gauge theories. Before we mention the results we have already got for simple toy models, we now discuss a possible application that displays a few of the subtleties one should be ready to face in the case of gauge theories.

A somehow artificial sign problem can be encountered by addressing the study of the Wilson action

$$
S_{G}[U]=\beta \sum_{m \in \Lambda} \sum_{\hat{\rho}<\hat{v}}\left[1-\frac{1}{2 N} \operatorname{Tr}\left(U_{\hat{\rho} \hat{v}}(m)+U_{\hat{\rho} \hat{v}}^{-1}(m)\right)\right]
$$

at complex values of the coupling $\beta$. Having in mind the construction of the thimble attached to the identity, one writes down the hessian

$$
\left.\nabla_{m, \hat{\rho}}^{b} \nabla_{n, \hat{\mu}}^{a} S_{G}[U]\right|_{U=\mathbb{I}}=\frac{\beta}{2 N} \delta^{a b}\left[2 d \delta_{n, m} \delta_{\hat{\mu}, \hat{\rho}}-\delta_{n, m}+\delta_{n+\hat{\mu}, m}+\delta_{n-\hat{\rho}, m}-\delta_{n+\hat{\mu}-\hat{\rho}, m}-\delta_{\hat{\mu}, \hat{\rho}} \sum_{\hat{v}}\left(\delta_{n+\hat{v}, m}+\delta_{n-\hat{v}, m}\right)\right]
$$

It is easy to realize that the spectrum displays not only the $(V-1)\left(N_{c}^{2}-1\right)$ zero modes we discussed above, but also extra $d\left(N_{c}^{2}-1\right)$ ones $^{2}$. This does not come as a surprise: torons have shown up. There is an extensive literature on the subject: the reader is referred to e.g. [7] and references therein for an introduction to the results we will mention in the following ${ }^{3}$. Already for the simple case $d=2$ and $N_{c}=2$ one gets a non-trivial case of study and since everything is known in this setting, we have the chance to validate the results of a thimble formulation.

Torons can be avoided by moving to a twisted action

$$
S_{G}[U]=\beta \sum_{P} f_{P}^{(t)}\left(U_{P}\right) \quad f_{P}^{(t)}\left(U_{P}\right)=\left\{f_{P}\left(z_{\hat{\mu} \hat{v}} U_{P}\right) \text { for } P \in R_{\hat{\mu} \hat{v}} ; f_{P}\left(U_{P}\right) \text { for } P \notin R_{\hat{\mu} \hat{v} \hat{v}}\right\}
$$

where $f_{P}\left(U_{P}\right)$ is the ordinary Wilson action density; $z_{\hat{\mu} \hat{v}}=z_{\hat{v} \hat{\mu}}^{-1}=\bar{z}_{\hat{v} \hat{\mu}}=e^{2 \pi i n_{\hat{\mu} \hat{v}} / N} \in Z_{N}$ is the twist tensor and $R_{\hat{\mu} \hat{v}}$ consists of a particular set of plaquettes. In our $d=2, N_{c}=2$ case $R_{\hat{\mu} \hat{v}}$ simply reduces to the plaquette which is named $P_{0}$ in figure 1. Minimum action configuration is now given by the so-called twist-eater. The construction of the latter starts with building the gauge tree on which the links can be gauged to unity. On the two ladders $L_{\hat{\mu}}$ and $L_{\hat{v}}$ links assume non-trivial

\footnotetext{
${ }^{2} \mathrm{~d}$ is the dimension the theory lives in.

${ }^{3}$ We thank A. Ramos for having pointed out ref [7] to us.
} 


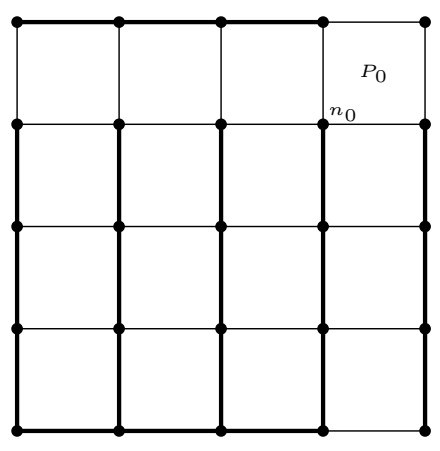

(a) Gauge tree

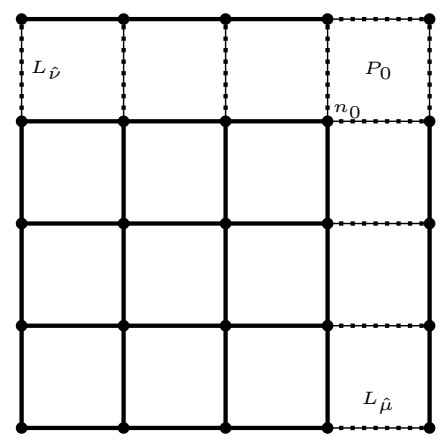

(b) Zero action configurations

Figure 1: The gauge tree construction for $d=2 . P_{0}$ is the only plaquette which enters the action with a non-trivial coefficient. $L_{\hat{\mu}}$ and $L_{\hat{v}}$ are the ladders on which links assume non-trivial values $G_{\hat{\mu}}$ and $G_{\hat{v}}$ respectively.

values $G_{\hat{\mu}}$ and $G_{\hat{v}}$ respectively. These values are decided by the the twisted commutation relation $G_{\hat{v}} G_{\hat{\mu}}=z_{\hat{\mu} \hat{v}} G_{\hat{\mu}} G_{\hat{v}}$ which puts to zero the contribution to the action coming from the plaquette $P_{0}$. It can be shown that the twist-eater is the global minimum, unaffected by torons. It is thus the critical point whose thimble one should naturally start taking into account.

\section{First applications: $\mathrm{SU}(\mathrm{N})$ toy models}

While the previous section referred to a problem $(d=2 S U(2)$ Yang-Mills theory) for which we only have plans, we have already worked out simple toy models like the $S U(N)$ one link models defined by

$$
S[U]=-\frac{\beta}{N} \operatorname{Tr}(U) \quad Z(\beta)=\int_{\mathrm{SU}(N)} \mathrm{d} U e^{\frac{\beta}{N} \operatorname{Tr}(U)}=\sum_{n=0}^{\infty} \frac{2 ! \cdots(N-1) !}{n ! \cdots(n+N-1) !}\left(\frac{\beta}{N}\right)^{N n}
$$

A natural observable is

$$
\langle\operatorname{Tr}(U)\rangle=\frac{1}{Z} \int_{\mathrm{SU}(N)} \mathrm{d} U \operatorname{Tr}(U) e^{\frac{\beta}{N} \operatorname{Tr}(U)}=N \frac{\partial}{\partial \beta} \ln Z
$$

Also in this case the sign problem is artificial (one takes complex values for $\beta$ ). It is important to point out that for $S U(N)$ one finds exactly $N$ critical points (the elements of the center $Z_{N}$ ). All of them give a contribution to the correct computation of results, as e.g. depicted in figure 2 in the case of $S U(3)$, where $|\beta|=5$ is kept fixed while $\arg \beta$ is varied.

\section{Conclusions}

Thimble regularization as a solution of the sign problem is still in its infancy and there is quite a long way to go before we can have it working for Lattice QCD. Nevertheless, a formulation for gauge theories is there and simple toy models have already been successfully worked out. 

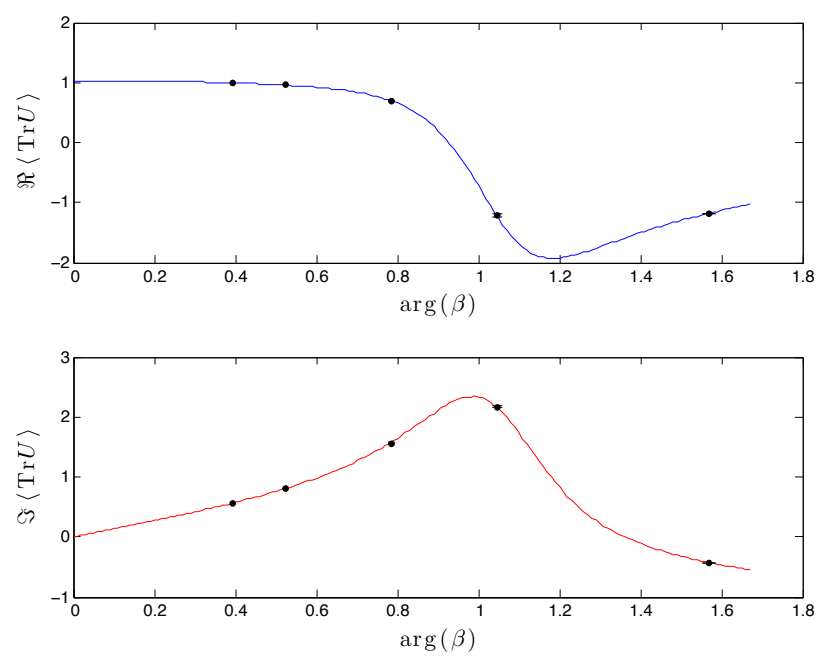

Figure 2: Computation of $\langle\operatorname{Tr}(U)\rangle$ at fixed $|\beta|=5$ for the $S U(3)$ one link model.

\section{References}

[1] E. Witten, Analytic Continuation Of Chern-Simons Theory, arXiv:1001.2933 [hep-th].

[2] M. Cristoforetti et al. [AuroraScience Collaboration], New approach to the sign problem in quantum field theories: High density QCD on a Lefschetz thimble, Phys. Rev. D 86 (2012) 074506 [arXiv:1205.3996 [hep-lat]].

[3] H. Fujii, D. Honda, M. Kato, Y. Kikukawa, S. Komatsu and T. Sano, Hybrid Monte Carlo on Lefschetz thimbles - A study of the residual sign problem, JHEP 1310 (2013) 147 [arXiv:1309.4371 [hep-lat]].

[4] F. Pham, Vanishing homologies and the $n$ variable saddle point method Proceedings of Symposia in Pure Mathematics 40 (1983) 321.

[5] M. Cristoforetti, F. Di Renzo, G. Eruzzi, A. Mukherjee, C. Schmidt, L. Scorzato and C. Torrero, An efficient method to compute the residual phase on a Lefschetz thimble, Phys. Rev. D 89 (2014) 11, 114505 [arXiv:1403.5637 [hep-lat]].

[6] M. F. Atiyah and R. Bott, The Yang-Mills equations over Riemann surfaces, Phil. Trans. Roy. Soc. Lond. A 308 (1982) 523.

[7] A. Gonzalez-Arroyo, Yang-Mills fields on the four-dimensional torus. Part 1.: Classical theory, In *Peniscola 1997, Advanced school on non-perturbative quantum field physics* 57-91 [hep-th/9807108]. 